\title{
ANALYSIS OF LiNK STATE RESOURCE RESERVATION PROTOCOL FOR CONGESTION MANAGEMENT IN SMART WEB HotSPOT ENVIRONMENTS
}

\author{
Anulika Okoye Joy ${ }^{1}$, K.C. Okafor ${ }^{2}$, I.A Godspower ${ }^{3}$ and O.A.Chinedu ${ }^{4}$ \\ ${ }^{1}$ Dept.of Electrical and Electronic Engineering, Chukwuemeka Odumegwu Ojukwu \\ University, (COOU) Uli, Anambra State, Nigeria \\ ${ }^{2}$ Dept. of Electrical and Electronic Engineering, Federal University of Technology \\ (FUTO), Owerri, Nigeria \\ ${ }^{3}$ Dept. of Computer Science, Nnamdi Azikiwe University (UNIZIK), Awka, Nigeria \\ ${ }^{4}$ Dept. of Electronic and Computer Engineering, Nnamdi Azikiwe University (UNIZIK), \\ Awka, Nigeria.
}

\begin{abstract}
With the wide spread of WiFi hotspots, concentrated traffic workload on Smart Web (SW) can slow down the network performance. This paper presents a congestion management strategy considering real time activities in today's smart web. With the SW context, cooperative packet recovery using resource reservation procedure for TCP flows was adapted for mitigating packet losses. This is to maintain data consistency between various access points of smart web hotspot. Using a real world scenario, it was confirmed that generic TCP cannot handle traffic congestion in a SW hotspot network. With TCP in scalable workload environments, continuous packet drops at the event of congestion remains obvious. This is unacceptable for mission critical domains. An enhanced Link State Resource Reservation Protocol (LSRSVP) which serves as dynamic feedback mechanism in smart web hotspots is presented. The contextual behaviour was contrasted with the generic TCP model. For the LS-RSVP, a simulation experiment for TCP connection between servers at the remote core layer and the access layer was carried out while using selected benchmark metrics. From the results, under realistic workloads, a steady-state throughput response was achieved by TCP LS-RSVP to about 3650Bits/secs compared with generic TCP plots in a previous study. Considering network service availability, this was found to be dependent on fault-tolerance of the hotspot network. From study, a high peak threshold of 0.009 (i.e. 90\%) was observed. This shows fairly acceptable service availability behaviour compared with the existing TCP schemes. For packet drop effects, an analysis on the network behaviour with respect to the LS-RSVP yielded a drop response of about 0.000106 bits/sec which is much lower compared with the case with generic TCP with over 0.38 bits/sec. The latency profile of average FTP download response was found to be 0.030secs, but with that of FTP upload response, this yielded about 0.028 sec. The results from the study demonstrate efficiency and optimality for realistic loads in Smart web contexts.
\end{abstract}

\section{KEYWORDS}

Hotspot Networks, TCP Flows, Traffic Congestion, Throughput, latency, Service Availability

\section{INTRODUCTION}

Smart web represents a disruptive paradigm shift in the generic wireless network architecture. It makes web services to serve both the real and virtual world users [1]. This type of web model is 
sensitive to targeted things/objects, application physical environments, or even situations, and respond proactively and intelligently. In Smart web, network congestion occurs when the resource demand is greater than the available computing resources, such as bandwidths of links, buffer memory space and CPU processing capacity, etc, at the intermediate nodes (such as routers, servers, storage, etc). However, Smart Congestion Control (SCC) is a modified perspective to existing congestion management schemes. This is because it focuses on using baseline feedback resource allocation to maintain an acceptable performance level on the demand of the available network resources. Therefore, a well detailed design is required to provide optimal service delivery under heavy realistic workload in smart web. Existing networks and methods for implementing context-aware web services on web 2 and 3 mainly enumerate different implementations corresponding to different attribute values of the network context in order to improve QoS. As things in the physical work merges with the virtual world, there is need to evolve a flexible congestion free network for smart web service of things.

This paper proposes a novel smart web services congestion control algorithm for end user context of things. When neglected, traffic oscillation that is highly disruptive will be evidenced in smart web networks. Consequently, TCP LS-RSVP is proposed to manage context of things in smart web services network. This implies that users can hardly notice any form of congestion in the network.

\section{Classical Theories on generic tCP Wlan Congestion ALGORITHM}

Before the advent of smart web internet, the current use of Wireless Local Area Networks (WLAN IEEE 802.11) hotspot for Internet access from wireless stations was dominated by downlink Transmission control Protocol (TCP) traffic. The TCP [2], is basically used to provide reliable communications on top of IP and Ethernet, as it employs loss-based congestion control algorithm. Packet loses are evidence with this type of algorithm as the rate of sending precisely controlled. Besides, point to point queuing delay can grow to the extreme maximum [3]. It known that the characteristics of TCP have not been sufficiently taken into account for data transport over WLANs [4] for smart web services.

Clearly, the congestion challenges found in hotspot infrastructures in homes, offices, and public areas has not been fully addressed [5] [6]. This has necessitated alternative approaches in smart computing.

To understand the issue of WLAN congestion, the important layers in IEEE 802.11 WLAN such as the Media Access Control (MAC) and Physical (PHY) layers have been defined by the IEEE 802.11 standard [6]. It has been observed that Flow-Aware WLANs [7] uses the Distributed Coordination Function (DCF) to coordinate channel access. However, in smart computing, TCP based Carrier Sense Multiple Access with Collision Avoidance (CSMA/CA) is still insufficient for a successful data frame transmission [4]. The duplication of acknowledgment functions at the MAC and transport layers, window size issues and other related overheads can significantly degrade the throughput performance in smart web computing. Hence, TCP scheme will hardly satisfy the QoS requirement of smart web in today's network.

In this paper, smart web refers to the online usage of intelligent and productive resources for effective computing. Examples include, chat services, download repositories (Google play store apps), On-demand storage/billing and Enterprise Resource Planning Systems (ERPs). There is 
need for a scheme that can eliminate the duplication of the two acknowledgements on the hotspot WLAN for smart web services. This scheme should produce a significant performance improvement, particularly in the infrastructure-mode WLAN running smart web services through an access point (AP). Congestion management in smart web computing will be explored through a proposed LS-RSVP.

\section{RELATED WORK}

\subsection{Congestion Management in Hotspots}

Rigorous study of literature reveals that efforts have been made to alleviate network congestion in wireless environments. The authors in [8], proposed a conceptual design model for high performance hotspot network infrastructure, (GRID WLAN) while investigating the effects of GRID WLAN access point buffer size distributions with respect to load intensities as well as fragmentation capacities.

The authors in [9] developed a rate estimation algorithm that uses symbol level combining for cooperative packet recovery in enterprise WLAN. Mobility susceptibility was discussed in [10] where node capacity and power consumption level contributes to TCP performance degradation often leading to packet losses.

The work in [11] focused on Delay Tolerant Networks (DTN) while proposing a buffer management policy for message controls by node buffers. Congestion behaviour an IEEE 802.11 based WLAN hosting web and shared file system was discussed in [12]. From their work, a queuing model, which provides the mean session delay in the presence of short-lived flows in the network, was developed. Their analysis accounts for the TCP delayed ACK option in the network. A TCP flow-aware wireless mesh network was presented in [13] while the authors in [14] presented a network performance optimization strategy. In [15], a novel transport protocol in the TCP/UDP family that provides a congestion-control was presented. This takes care of the flow of unreliable datagram. The works in [16], [17], [18] discussed on WLANs networks with related TCP congestion schemes.

From the review studies, a link state algorithm considering a two stage BSS model and other WLAN infrastructural (offering FTP and HTTP) services for a high density network is yet to be developed. This will offer a useful guild to developing Internet of Things (IoT) network QoS evaluation. This paper, will use a real testbed as a requirement for the study of generic TCP congestion control scheme in a wireless network scenario. It is argued that a well designed network testbed that provides stable data rates, excellent buffer management, and wireless packet recycling with a fair level of QoS services for all traffic sources must be maintained.

\subsection{Limitations of Existing Schemes}

Based on the analysis of existing research efforts, the following observations are highlighted:

- Existing works failed to account for explicit resource reservation for TCP flows thereby avoiding packet losses in heavily congested environment while still allowing nodes to gain fair access to the channel.

- Existing works still limits their discussion to frame transmissions at $1 \mathrm{Mbps}$ and $11 \mathrm{Mbps}$ for all congestion levels. This is unacceptable for current high density hotspot implementations for smart web services. 
- At high congestion levels, details on successful transmission of large frames/packets has not been fully addressed

- The issue of quality of service (QoS) only considered connection oriented protocol-TCP and not connectionless oriented protocols -UDP

- The feedback algorithms in existing works induce over 30\% network delay, thereby creating a non responsive network.

The deficiencies in the IEEE 802.11 protocols considering TCP flow implementations demands for an improved strategy. This research will empirically analyse a heavily-congested wireless network using LS-RSVP. This will be carried out to offer significant insight into the behaviour of SW network. With the insight, this can be utilized to design better systems and protocols

\section{RESEARCH METHODOLOGY}

The methodology used in this work follows the decomposition of traffic bucket for congestion management in a selected reference testbed. Using a real world testbed (University of Nigeria, ICT datacenter hotspot) that supports interactive traffic such as Telnet, voice over IP, text data, email, http etc, Ethereal Wireshark was used to observe TCP packet drops with increased queues (network users) carrying out large packet LAN-to-LAN FTP transfers, browsing, downloading, etc. This becomes obvious as they are queue on slower links. The approach here is to investigate on a traditional TCP-IP congestion scheme on the basis of QoS features such as delay, throughput, jitter, etc on slower-speed links. Fig. 1 is a representative framework of a traditional TCP-IP congestion control mechanism depicted in UNN as a case study.

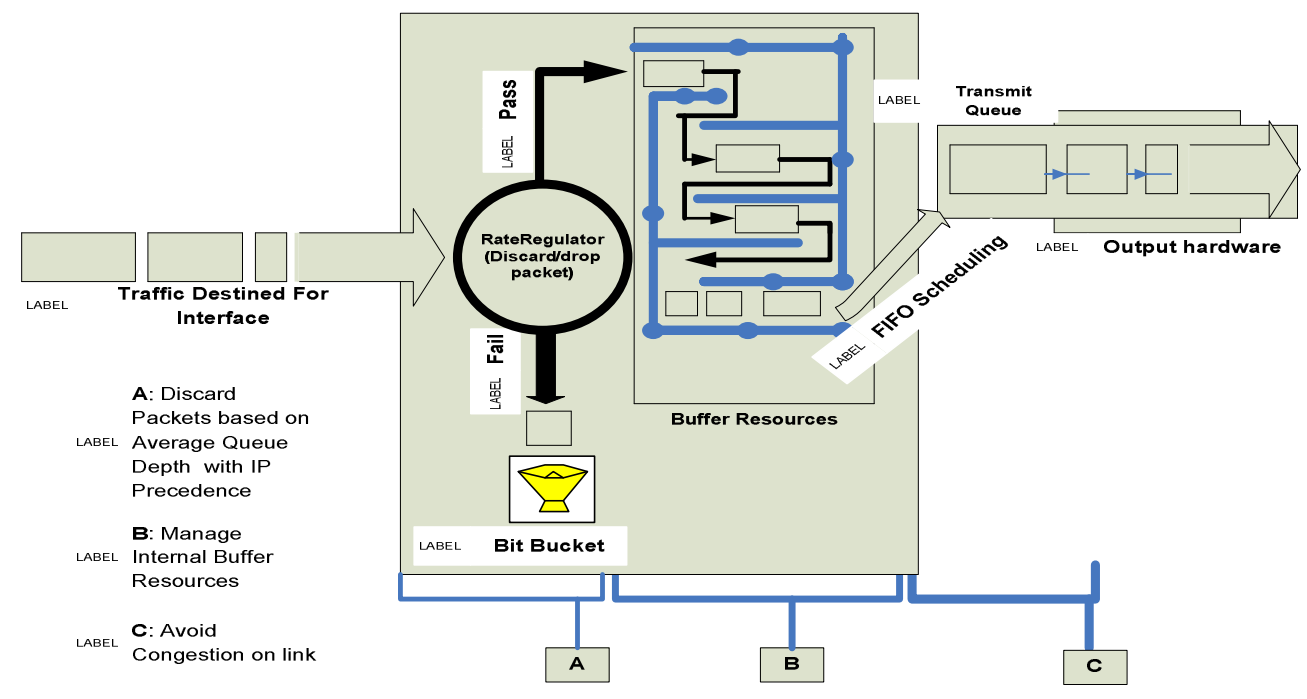

Figure 1. A model for traditional TCP-IP Congestion Control Mechanism

Having identified the challenges found in Fig.1, this work then developed a replacement model shown in Fig.2. In this case, the dataset behaviour of ethereal Wireshack in the production network running interactive traffic such as Telnet, Voice over IP, email, http, etc was leveraged for the study. With this replacement framework, services will be less susceptible to packet drops even at high realistic loads. With the resource reservation, latency and jitter will be minimized 
particularly, when the network processes large packets (for example, LAN-to-LAN FTP transfers traversing a WAN link). This suits the hotpot scenario where down time is dreaded. This framework offers a more viable congestion avoidance scheme within the context of smart computing in the hotspot infrastructure. As shown in Fig.2, the rate regulator controls incoming traffic as well as utilizes the buffer resources for queuing. FIFO scheduling is then used to output services after some form of processing.

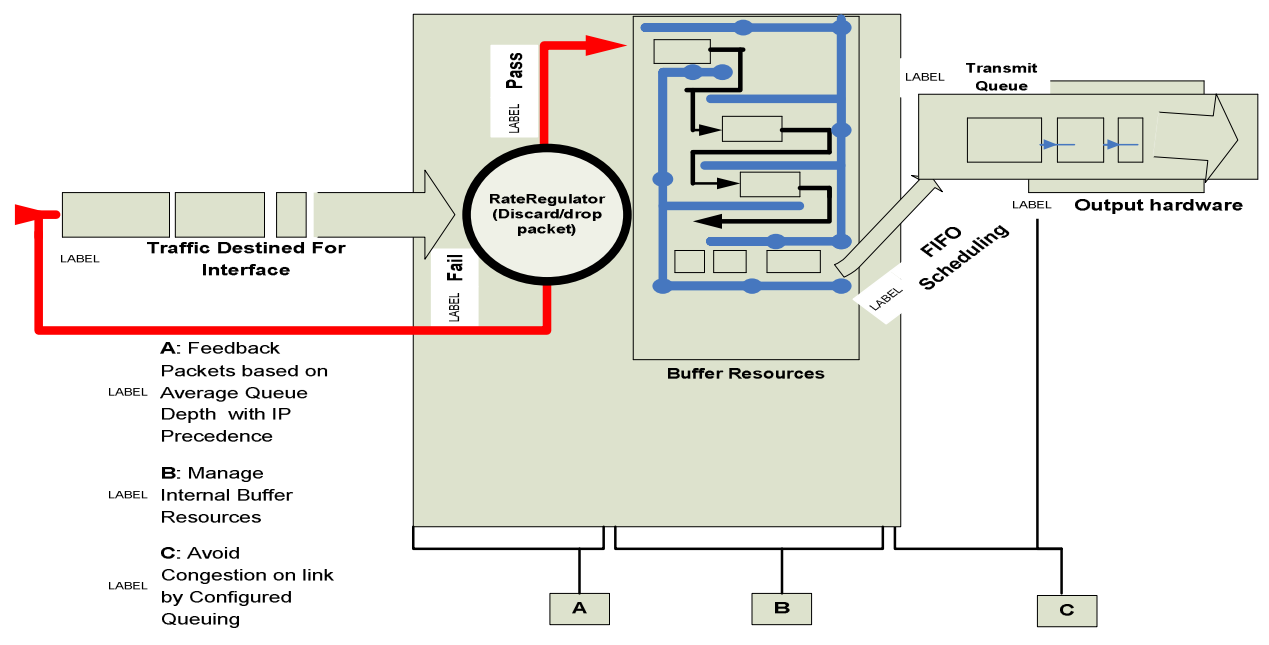

Figure 2. An Improved TCP-IP Congestion Control Mechanism based on LS-RSVP

\subsection{Algorithm for Link State Resource Reservation Setup Mechanism}

Various works on congestion management has been studied previously with some identified the research gaps. This section focused on formulating the algorithm of smart web LS-RSVP considering the entire network architecture and some QoS metrics for evaluations. The Riverbed $\mathrm{C}++$ Modeller simulator was used for the configuration and implementation. The profile and application palettes were set up for all the intended services for a hotspot scenario. These services occur on three levels: Application-RSVP interface process level, RSVP process level, Traffic Control process levels. RSVP is a hop-by-hop feedback QoS signaling protocol. This means that LS-RSVP messages are transmitted from a source node to a destination sink using RSVP-aware data path. The LS-RSVP approach handles traffic flow a sender to a sink node as logically independent of other users on the network. Meanwhile, a reservation is made for each transmission cycle. The flow in RSVP is a form of full duplex communication where the reservations for traffic users can be made from any or both directions. The reservation setup mechanism is shown in the algorithm in Table 1. 
Table 1: LS-RVSP for Smart Web Congestion Control
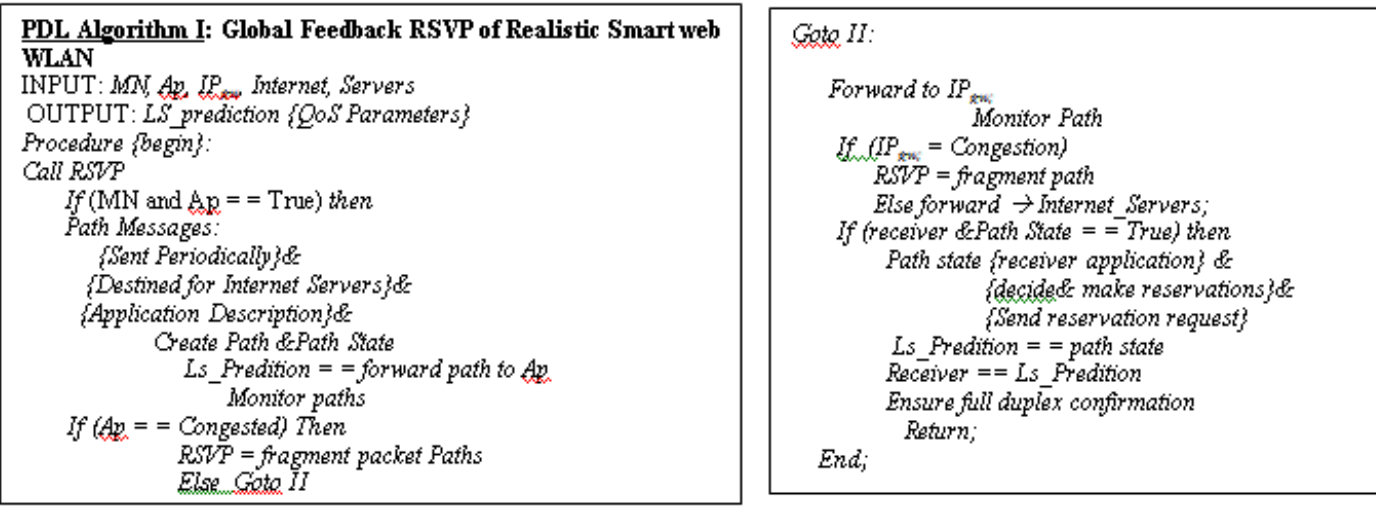

From the algorithm in Table 1, a unicast or multicast flow compel a sender node to be aware of the characteristics of the sink node before instantiating the LS-RSVP. There could be regular or periodic updates using RSVP messages. Every request and acknowledgement including the state of the network resource like link bandwidth, buffer sizes, etc are satisfied before any form of transmission can take place. The sink will always request for a notification about the status of the connecting network devices. An affirmative confirmation implies that the network resource conditions are satisfied. In this case, feedback signalling is used to accept the incoming or outgoing messages. Link prediction is found useful in this regard. If the reservation is successful, a Resv state is created in the data path as well as in the entire network. In each instance, when the congestion state exceeds the device holding capacity, the feedback path as depicted in Fig. 2 is instantiated. In the implementation, the LS-RSVP is achieved in the simulation design. The supported services the network context whose process runs only in IP-enabled nodes include the following applications: Database, Email, FTP, Remote Login, Video Conferencing (for both unicast and multicast sessions), and Voice (for both unicast and multicast sessions). The merits of LS-RSVP are discussed below.

\subsection{Advantages of Smart Web LS-RSVP Algorithm}

While the proposed algorithm LS-RSVP has the ability to adjust the transmission rate constraints based on congestion feedback with resource reservations, it still has constraints on how this adaptation can be performed to minimize unpredictable QoS issues. Thus, it will need some control over the short-term dynamics of congestion disturbances while being fair to other traffic on medium timescales. The merits of the proposed algorithm include:

i. Low per-packet overhead. Here, Smart web service communication will achieve lower delay in terms of response time with high throughput. Protocol overhead does not expand the packets unduly leading to fast response times or latency.

ii. Middlebox Traversal: TCP-Friendly Rate Control (TFRC) and TCP layer 4dynamic modulation feedbacks are a congestion control mechanisms that adjusts its sending rate more smoothly than the generic TCP does, while maintaining long-term fair bandwidth sharing with TCP.

iii. QoS fairness and Stability: The scheme offers a rate based feedback that is light weighted while supporting congestion control. Clearly, QoS fairness and stability are achieved by instantaneous feedback negotiation in the Fig. 2 . 


\section{PERFORMANCE EVALUATIONS}

\subsection{Simulation Testbed}

To demonstrate the network architecture of the hotspot environment running LS-RSVP algorithm implementation, a simulation testbed experiment was built with OPNET IT Guru 9.1 (now riverbed modeller 17.5). The LS-RSVP algorithm is then configured in the OPNET engine. This consists of a large scale remote datacenter servers running Http and web services. Ten subnet sites were created which houses 100 smart mobile client nodes. Also, ten access points were used as their respective base stations. Then an IP gateway router was used to connect all the smart web users to the IP cloud. The TCP LS-RSVP algorithm was configured in the trace files for all mobile nodes including the APs in six basic configuration scenarios. In this case, load variation was made from five users to forty users in the ten subnets sites. The subnet sites and IP gateway cloud emulates the smart web WAN link with the desired throughput, delay/latency, service availability, packet data drop, FTP/HTTP latency profiles. Now, Fig 2 was implemented in OPNET IT Guru which was used to generate the parameters for the case scenario in the simulations. Traffic attributes for the smart web WLAN were configured in the simulation engine. The runtime environment attribute were as follows in the OPNET simulator. In the characterization of the experimental and simulation testbed, the configured with values in the OPNET engine was leveraged. Simulation duration of 120 minutes for each scenario was used; Link propagation delay was set at 0.5 millisecons. The switch output buffer has 100packets with an update interval of 5000events. Various TCP variants were also enabled. Running the test bed, the following metrics were measured viz: throughput, Delay/latency, service availability, packet data drop, FTP/HTTP latency profiles, via template scenario of Fig. 3 which shows the validation testbed while Fig. 4 and 5 shows the link State RSVP successful simulation run/compilation for smart web context following Table 2

Table 2: Smart Web -WLAN Simulation Parameter Table for TCP LS-RSVP

\begin{tabular}{|c|c|c|c|c|c|c|c|}
\hline $\begin{array}{l}\text { TCP } \\
\text { TYPE }\end{array}$ & $\begin{array}{l}\text { Smart Web WLAN } \\
\text { Parameters }\end{array}$ & $\begin{array}{l}\text { 5 Sources. Smart } \\
\text { WLAN BUFFER- } \\
64 \mathrm{~K}\end{array}$ & $\begin{array}{l}10 \text { Sources } \\
\text { WLAN } \\
\text { BUFFER- } \\
128 \mathrm{~K}\end{array}$ & $\begin{array}{l}15 \text { Sources } \\
\text { WLAN } \\
\text { BUFFER- } \\
256 \mathrm{~K}\end{array}$ & $\begin{array}{l}20 \text { Sources } \\
\text { WLAN } \\
\text { BUFFER- } \\
512 \mathrm{~K}\end{array}$ & $\begin{array}{l}30 \text { Sources } \\
\text { WLAN } \\
\text { BUFFER- } \\
1024 \mathrm{~K}\end{array}$ & $\begin{array}{l}40 \text { Sources } \\
\text { WLAN } \\
\text { BUFFER- } \\
2048 \mathrm{~K}\end{array}$ \\
\hline \multirow[t]{11}{*}{$\begin{array}{l}\text { Proposed } \\
\text { LS-RSVP } \\
\end{array}$} & RTS-Threshold (Bytes) & 256 Bytes & 256Bytes & 256Bytes & 256Bytes & 256Bytes & 256Bytes \\
\hline & $\begin{array}{l}\begin{array}{l}\text { Fragmentation Threshold } \\
\text { (Bytes) }\end{array} \\
\end{array}$ & 256 Bytes & 512Bytes & 1024Bytes & 2048Bytes & 4096Bytes & 8192Bytes \\
\hline & Data Rate (bps) & 54 & 54 & 54 & 54 & 54 & 54 \\
\hline & Physical Characteristics & DSSS (D3S) & DSSS & DSSS & DSSS & DSSS (D3S) & $\begin{array}{l}\text { DSSS } \\
\text { (D3S) }\end{array}$ \\
\hline & $\begin{array}{ll}\begin{array}{l}\text { Packet-Reception } \\
\text { Threshold(W) }\end{array} & \text { Power } \\
\end{array}$ & $7.33 \mathrm{e}-14$ & $7.33 \mathrm{e}-14$ & $7.33 \mathrm{e}-14$ & $7.33 \mathrm{e}-14$ & $7.33 \mathrm{e}-14$ & $7.33 \mathrm{e}-14$ \\
\hline & Short Retry Limit & 7 & 7 & 7 & 7 & 7 & 7 \\
\hline & Long Retry Limit & 4 & 4 & 4 & 4 & 4 & 4 \\
\hline & AP Functionality & Active & Active & Active & Active & Active & Active \\
\hline & Buffer Size (bits) & $64 \mathrm{~K}$ & $128 \mathrm{k}$ & $256 \mathrm{k}$ & $512 \mathrm{k}$ & $1024 \mathrm{~K}$ & $2048 \mathrm{~K}$ \\
\hline & Max-Receive Lifetime (Sec) & 0.5 & 0.5 & 0.5 & 0.5 & 0.5 & 0.5 \\
\hline & Large-Packet Processing & Predicted & Predicted & Predicted & Predicted & Predicted & Predicted \\
\hline
\end{tabular}


International Journal of Grid Computing \& Applications (IJGCA) Vol.7, No.2, June 2016

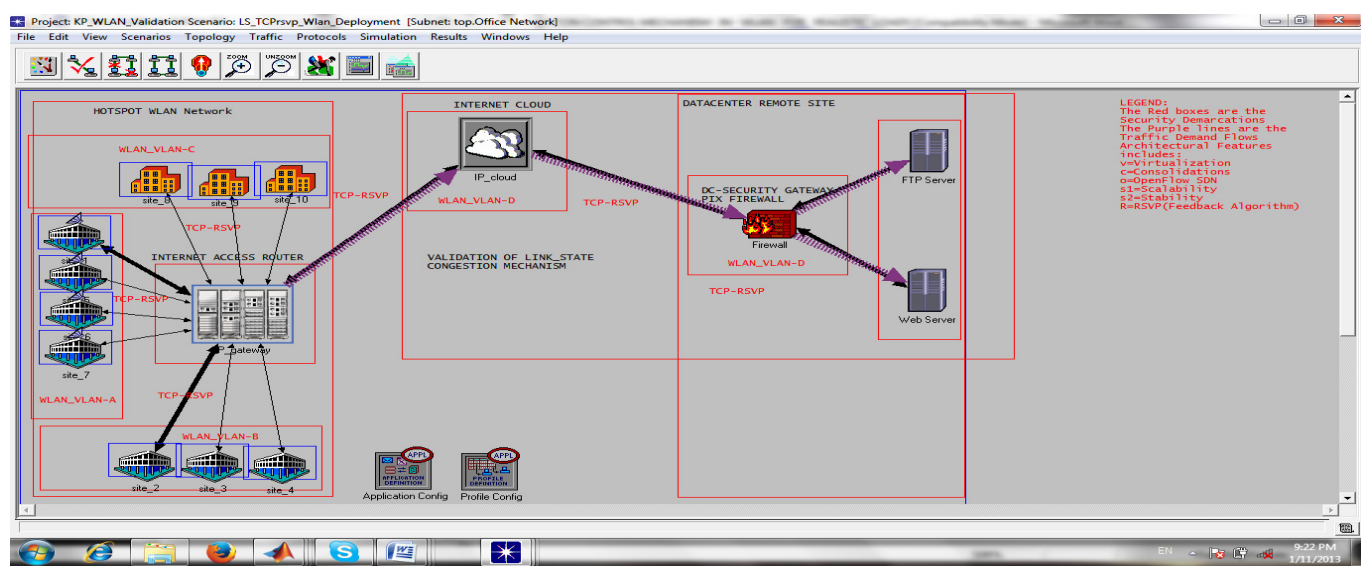

Figure 3. Validation testbed with Smart web WLAN Subnet 0 to subnet 10.

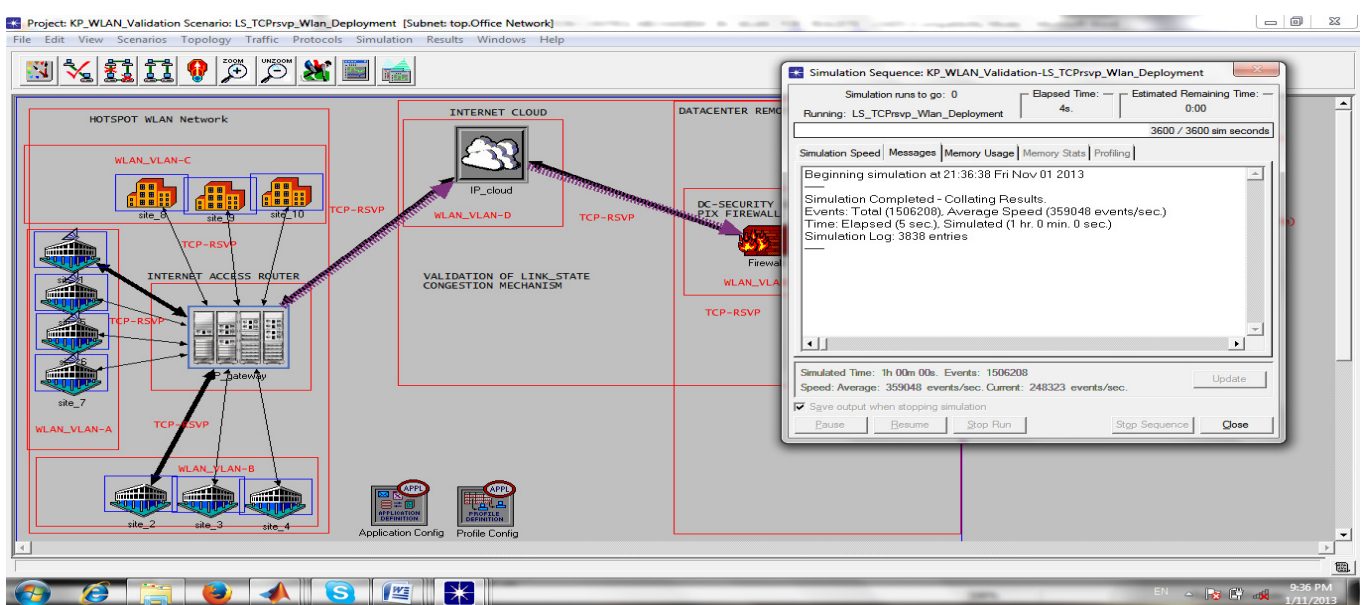

Figure 4. Link State RSVP successful simulation Run

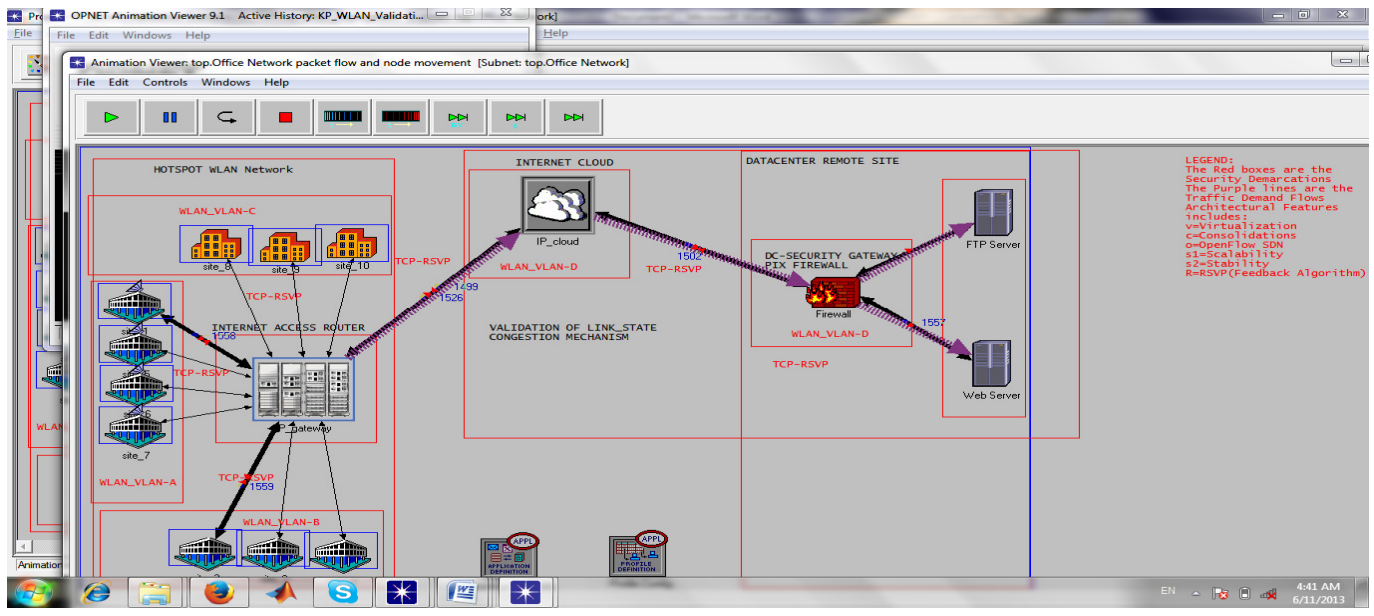

Figure 5. Hotspot WLAN Realistic Load Traffic Animation Scenario 


\subsection{Analysis of Results}

In this work, an evaluation on LS-RSVP TCP variant was carried out for the smart web network infrastructure using the selected QoS metrics. Essentially, the smart web QoS explains the overall performance of the network as perceived by the end users. The analysis in context focused on using a quantitative approach to analyze the QoS behaviour in the proposed network. These are briefly discussed below.

\section{i. Throughput QoS Response}

Fig. 6 illustrates the steady-state throughput response achieved by TCP LS-RSVP algorithm under realistic loads. Interestingly, the algorithm shows a progressive line trend over a long duration of time with peak throughput value of over 3650Bits/secs. This value is relatively higher than most TCP schemes. This is due to its connection oriented behavior leading to non degradation of the network as user density increases under realistic load conditions. In high performance computing networks, this type of behaviour will facilitate network scalability with dynamic services automation on the cluster server backbone. The trade-off could be cost of maintenance which is currently addressed by bare-metal virtualization and dynamic over allocation schemes. This could be a very good reason for consolidation of servers and other logical resources in smart web infrastructures.

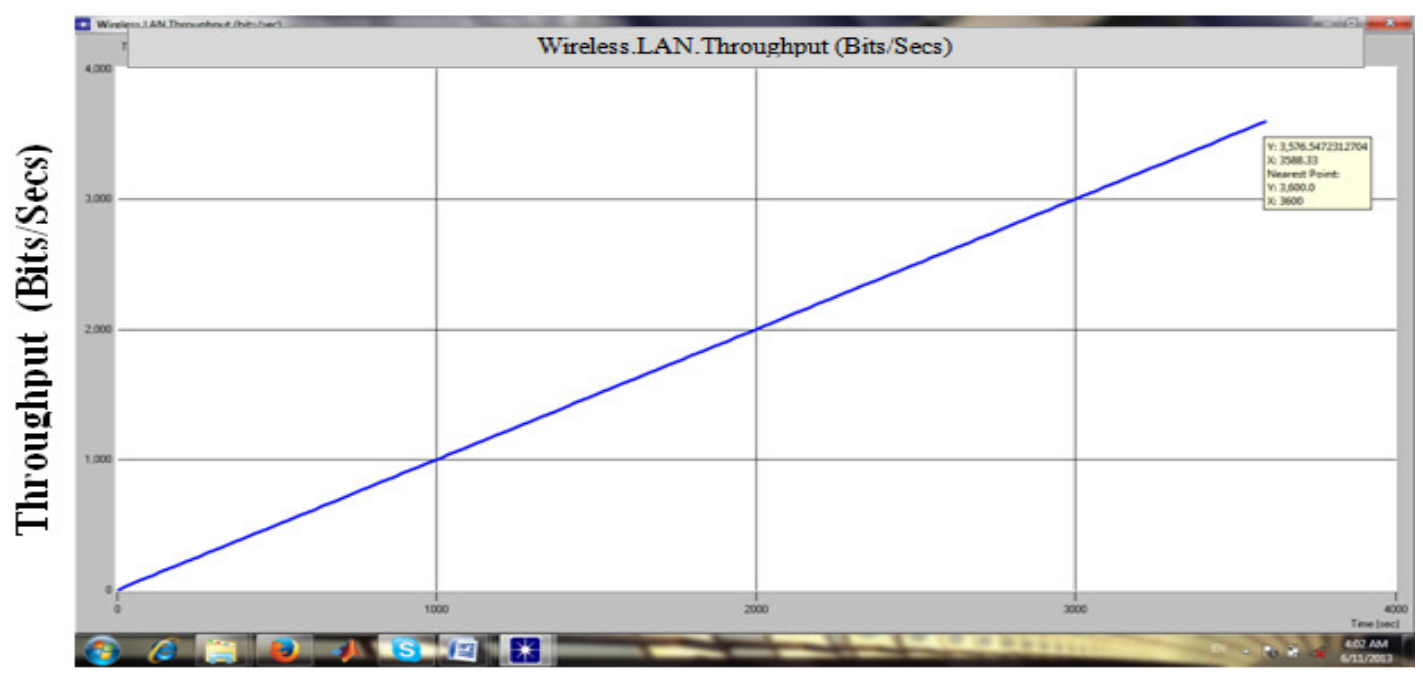

Figure 6: LS-RSVP Throughput Plot

\section{ii. Delay QoS Response}

Fig.7 shows the latency plot of LS-RSVP under realistic load in smart web environment. It represents the performance seen by the smart web users in the network. It was observed that latency trend curve began from zero and normalized at 0.0004s for the WLAN realistic load scenario. Essentially, the proposed LS-RSVP showed a comparative latency response when with generic TCP. It maintained a steady rate of about $0.0004 \mathrm{~s}$ relative to generic TCP control scheme. The feedback algorithm of Fig. 2 scenario enabled fast initialization for incremental users on the network under peak congestion states. A delay or latency value of $0.0004 \mathrm{~s}$ literally implies a very 
fast network. This will directly impact on the network throughput, thereby enhancing users experience on the smart web.

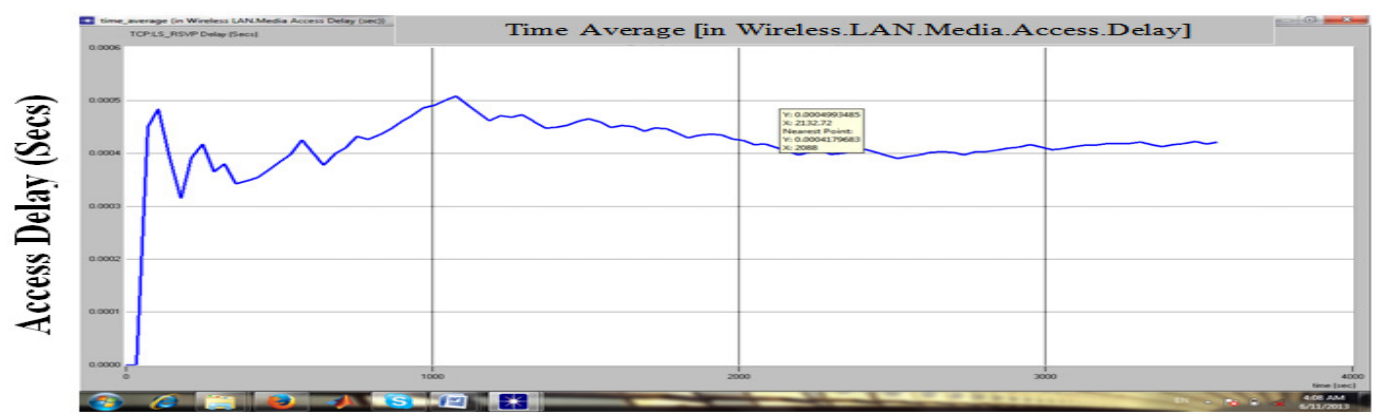

Figure 7: LS-RSVP Delay Plot

\section{iii. Network Service Availability QoS Analysis}

Fig. 8 depicts the network service availability response plot of the congestion state. The network availability is dependent on fault-tolerance of the bulk arrival and bulk service policy in smart web hotspot network. A high uptime or service availability depicts an acceptable operational performance. By eradicating single point of failures through redundancy, introducing efficient crossover as well as failure detection systems, reliability could be derived.

To study the performance under link failures (downtime), the link interfaces were manually unplugged in some selected hotspot sites and then plugged in at time $42 \mathrm{~s}$ in the simulation panel. Also few backend servers [1 to 2] were shut down at time 104s in order to assess the impact of congestion modes of LS-RSVP. Interestingly, path convergence was observed. The LS-RSVP traffic was maintained as well and the CPU utilizations are about $40 \%$, and $45 \%$, for senders, and receivers, respectively. This is a different scenario for generic TCP based networks. In context, this work makes two observations from the experiment. First, the smart web WLAN is a very resilient to congestion failures. The network throughput is recovered to the best value after only a few seconds compared to generic TCP congestion mechanism.

Second, the smart WLAN implementation detects link failures and node failures much faster than generic TCP algorithm because it uses medium sensing network capacity, with its fault tolerant and suppression routing algorithm.

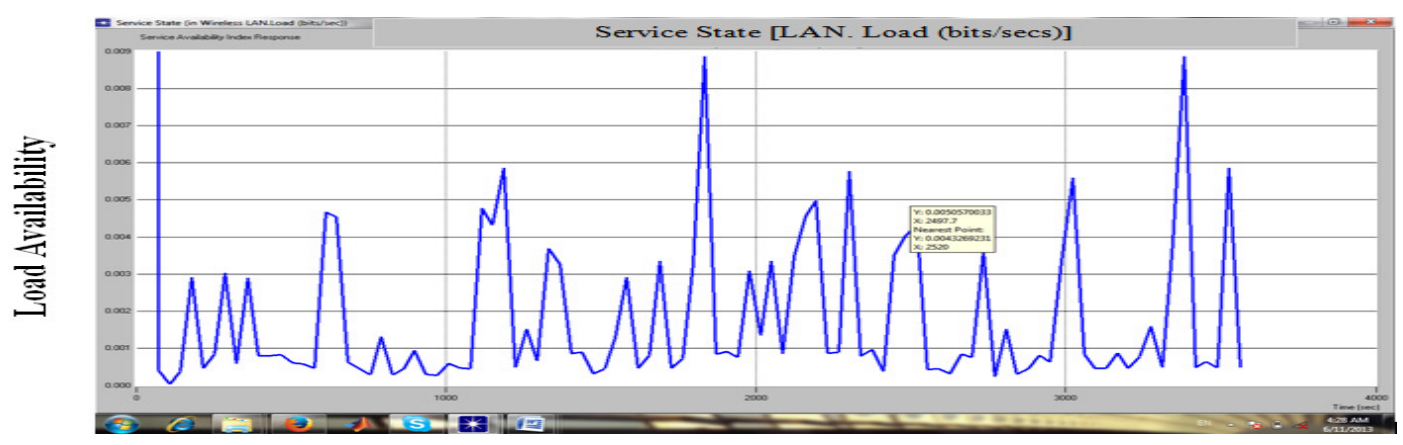

Figure 8: Network Service Availability Response Plot 
Clearly, from the plot in Fig. 8, it was showed that the proposed LS-RSVP hotspot smart WLAN had relatively more service availability compared with the existing TCP WLAN model. For the flow traffic, the peak threshold occurred at 0.009 . i.e. $90 \%$ which is fairly acceptable. In the production environment, this must be improved upto $99.99999 \%$ which will amount to $0.0864 \mathrm{~ms}$ downtime per day. When the system is up, smart web services will remain available. The network outage could only occur when the redundant components fail as a result of human or machine error. In this regard, effective load balancing and failover are active redundant schemes used in smart web cloud environment to achieve zero performance degradation or even outage of any kind.

\section{iv. Packet Drop Effects}

In this case, an analysis on the network behavior with respect to the LS-RSVP algorithm discussed previously was carried out. Fig. 9 shows the end-to-end packet drop result of the LSRSVP algorithm for two-tier topology of Fig.3 topology reflecting the discussion carried out in this study. From the result, packet drop response shows a great dissimilarity with previous study. As observed in the plot, packet drop response was observed to be about $0.000106 \mathrm{bits} / \mathrm{sec}$ which is much lower compared with the case with generic TCP with over $0.38 \mathrm{bits} / \mathrm{sec}$. At the event of congestion, its feedback loop recycles packets avoiding congestion scenarios.

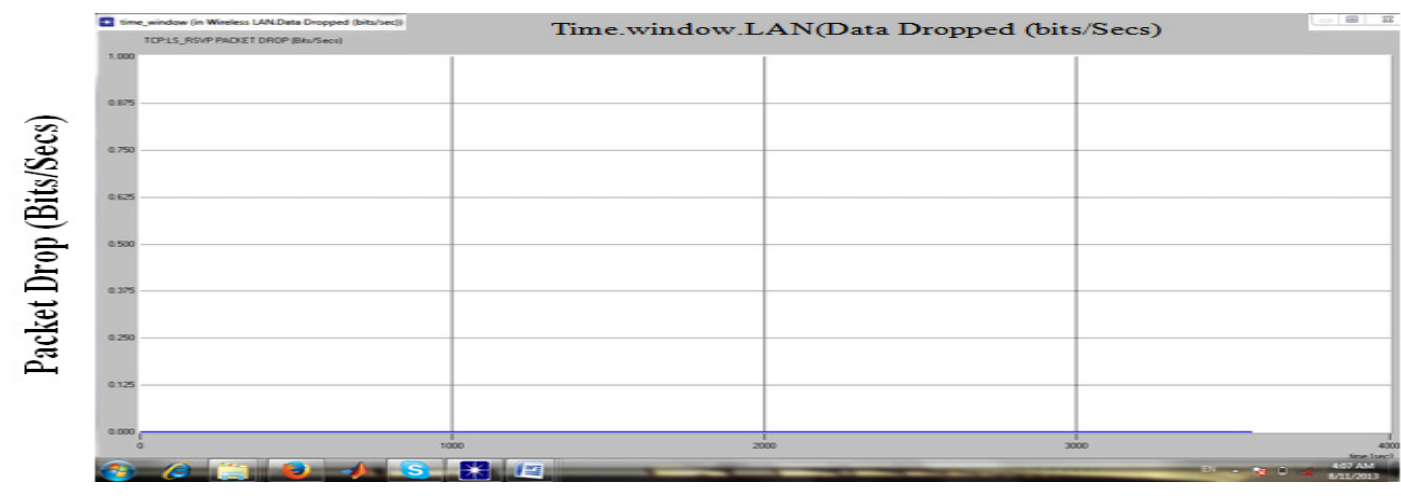

Figure 9: Packet Drop Response (Bits/Secs)

\section{v. FTP, HTTP Latency Profiles}

Fig. 10 shows the latency profile of average FTP download response to be 0.030 secs with that of FTP upload response is about $0.028 \mathrm{sec}$. Fig. 11 shows the latency profile of average HTTP page response to be 0.0389 secs. From the context of smart web WLAN, these values demonstrate efficiency and optimality for realistic loads. 
International Journal of Grid Computing \& Applications (IJGCA) Vol.7, No.2, June 2016

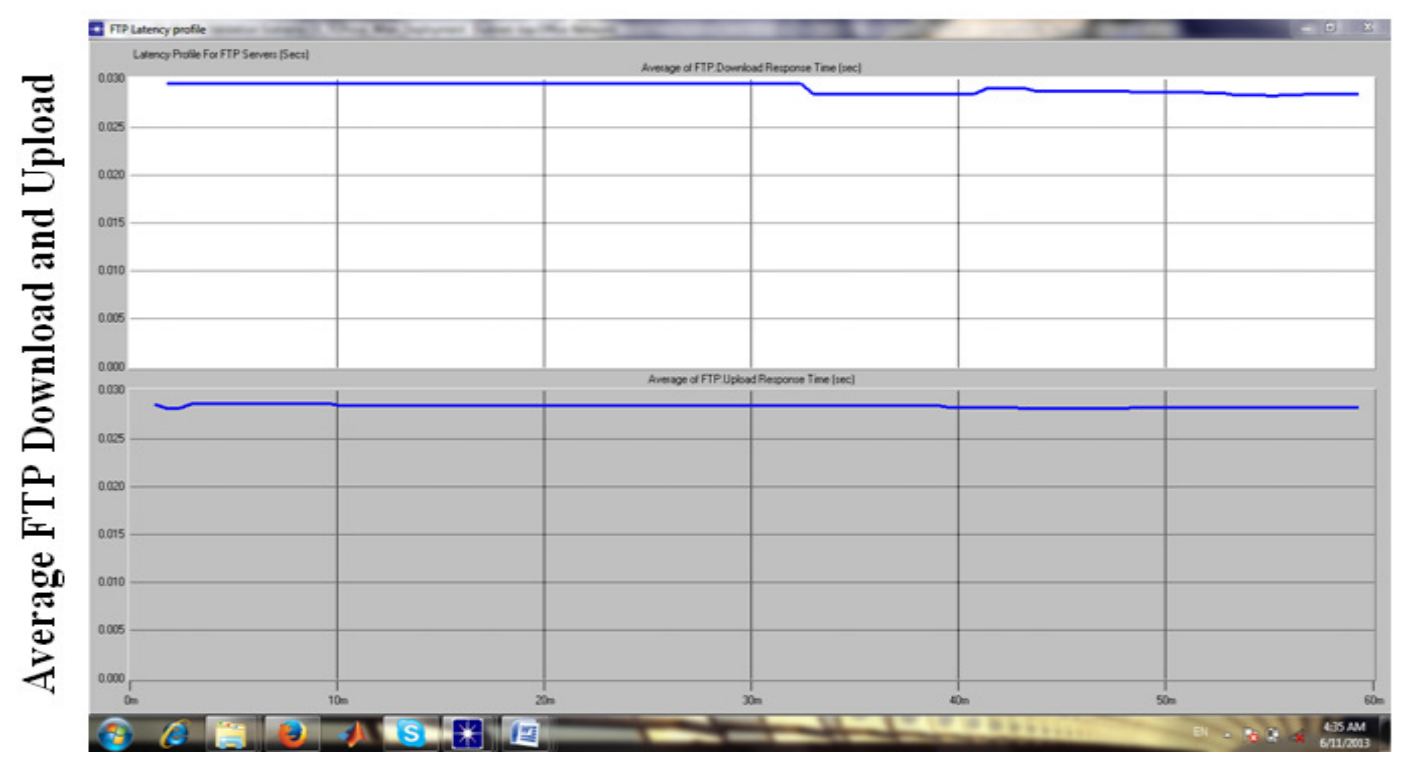

Figure 10: FTP Latency Profile

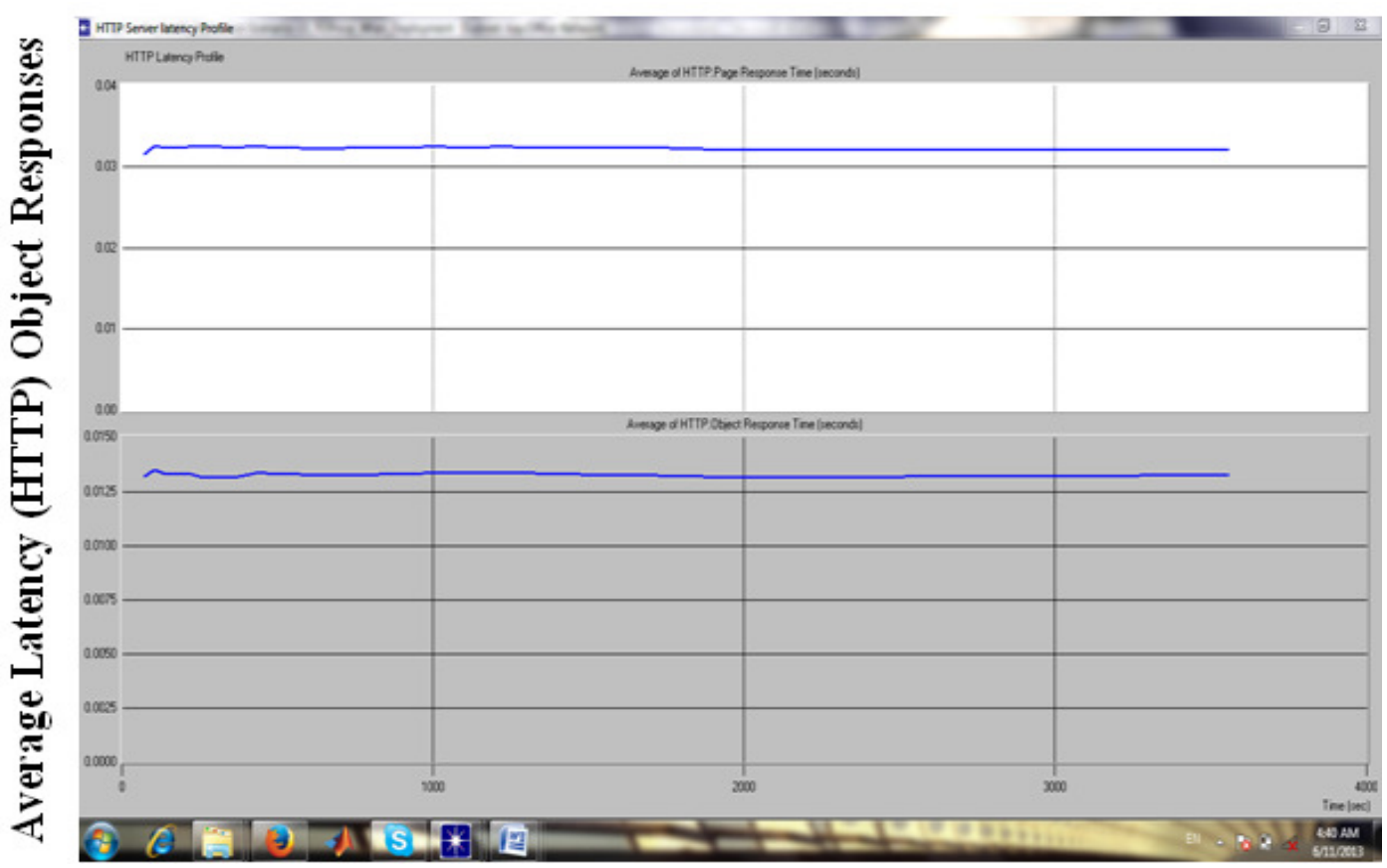

Figure 11: HTTP Latency Profile

In summary, the proposed link state algorithm (LS-RSVP) which is based on a two stage BSS model and other WLAN infrastructures have been described. In the network, a consideration for FTP and HTTP services in a high density network has been discussed in relation to smart web networks. This was carried out after considering a real life testbed that have limitations based on generic TCP congestion control scheme for a wireless network scenario in an earlier work [8]. A 
well design network testbed that provides stable data rates, excellent buffer management for APs and wireless devices with packet recycling and a fair level of Quality of Services for all traffic sources was achieved for smart web environment. The framework for the proposed link state TCP algorithm implementation was realized with a trace file in Riverbed C++ Modeller prediction model. The essential parameters in this research includes viz: Throughput, delay, throughput, service availability, utilization.

\section{CONCLUSIONS}

In this paper, an improved congestion management scheme was introduced in smart hotspot networks considering realistic loads. The use LS-RSVP for effective QoS provisioning was discussed while outlining its merits. The limitations of existing works were highlighted while justifying our initial proposal, via a simulation study for the selected metrics. With existing TCP scheme, smart web networks will continually experience congestion under realistic loads. The proposed LS-RSVP uses dynamic feedback mechanism in any given hotspots environment to improve QoS. Besides its can dynamically adapt to extended service set designs. From the results, under realistic loads, the steady-state throughput response achieved by TCP LS-RSVP algorithm was observed to about 3500 packet/bits compared with TCP plots in our earlier empirical study. The latency plot of LS-RSVP under realistic load maintained a steady rate of about $0.0004 \mathrm{~s}$ which makes network process automation easily achievable compared with existing TCP schemes. Considering network service availability, this was observed to be dependent on fault-tolerance of the hotspot network (i.e. its redundancy design). In this regard, a fairly observable peak threshold was achieved. This opined to be a reasonable service availability compared with the existing TCP WLAN model. For packet drop effects, an analysis on the network behavior with respect to the LS-RSVP algorithm yielded a lower packet drop response of compared with the case with generic TCP. Further, it was showed that the latency profile of average FTP download and FTP upload responses are satisfactory for smart web services provisioning. Future work will present the validation and empirical results from a mathematical model of the system testbed.

\section{ACKNOWLEDGEMENTS}

We wish to thank the University of Nigeria Nsukka ICT innovation center network administrators and Kswitche Research \& Consults Groups for their informed technical inputs and supports.

\section{REFERENCES}

[1] J.He, Y.Zhang, G.Huang, J.Cao, ( 2012), "A smart web service based on the context of things", Journal ACM Transactions on Intenet Technology(TOIT), Vol.11, Issue 3, New york, USA.

[2] S.Floyd, et.al, (1999) "The NewReno Modification to TCP's fast Recovery Algorithm", RFC 2582,IETF.

[3] H.Shimonishi, J.Higuch, T.Yoshikawa, and Atsushi Iwata, "A Congestion Control Algorithm For DataCenter Area Communications,

[4] Qixiang Pang, Soung C. Liew, Victor C.M. Leung, "Performance Improvement of 802.11 Wireless Access Network with TCP ACK Agent and Auto-Zoom Backoff Algorithm.

[ 5] M. S. Gast, (2002), “ 802.11 Wireless Networks: The Definitive Guides”, O'Reilly.

[6] IEEE Standard for Wireless LAN Medium Access Control (MAC) and Physical Layer (PHY) Specifications, (1999), ISO/IEC 8802-11:1999E)..

[ 7] S. Oueslati and J. Roberts, (2005), "A new direction for quality of service: Flow-aware Networking," in NGI 2005, Rome, Italy. 
International Journal of Grid Computing \& Applications (IJGCA) Vol.7, No.2, June 2016

[ 8] Udeze C. C, Okafor K.C, H. C Inyiama, C. C. Okezie, (2012) “ A Conceptual Design Model for High Performance Hotspot Network Infrastructure (GRID WLAN)", (IJACSA) International Journal of Advanced Computer Science and Applications, Vol. 3, No. 1, Pp.93-99 .

[ 9] Mahanth Gowda, Souvik Sen,Romit Roy Choudhury, Sung-Ju Lee, "Cooperative Packet Recovery in Enterprise WLANs"

[ 10] Haniza N., Zulkiflee M., Abdul S.Shibghatullah, Shahrin S., Congestive Loss in Wireless Ad hoc Network-Network Performance Analysis"World of Computer Science and Information Technology Journal (WCSIT) ISSN: 2221-0741 Vol. 1, No. 6, 269-273, 201. Pp. 269-273

[11] Amir Krifa, Chadi Barakat, Thrasyvoulos Spyropoulos, (2008),“ "Optimal Buffer Management Policies for Delay Tolerant Networks" In Proc. 2nd Conf. San fransico.

[12] Daniele Miorandi a,Arzad A. Kherani b, Eitan Altman,(2006),"A queuing model for HTTP traffic over IEEE 802.11 WLANs, Computer Networks50, 63-79.

[13] Juan J. Galvez, Pedro M. Ruiz, Antonio F. Gomez Skarmeta, "TCP flow-aware Channel ReAssignment in Multi-Radio Multi-Channel Wireless Mesh Networks"

[14] Amit P. Jardosh Krishna N. Ramachandran Kevin C. Almeroth Elizabeth M. Belding-Royer, "Understanding Congestion in IEEE 802.11b Wireless Networks",

[15] Eddie Kohler, Mark Handley, Sally Floyd, "Designing DCCP: Congestion Control Without Reliability.

[16] Ghaida A. AL-Suhail, Turki Y. Abdallah, and Omar Majid, (2012), "Fuzzy-Logic Adaptive Queuing For A Heuristic TCP Performance In Mobile Wireless Networks", International Journal of Computer Networks \& Communications (IJCNC) Vol.4, No.3, Pp. 191-205.

[ 17] Welzl M. "Network Congestion Control: Managing Internet Traffic," 282 pages, July 2005.

[ 18] J. Widmer, C. Boutoemans, J. Y. Boudec, (2004), "End-to-End Congestion Control for TCP Friendly Flows with Variable Packet Size", ACM SIGCOMM 2004.

[ 19 ]J.C. Ochi, C.O. Ohaneme, A.C.O. Azubog,(2015), "Development of an Intelligent Fuzzy-Based Algorithm for Data Congestion Management Scheme in Wireless LAN", International Journal of Innovative Technology and Exploring Engineering (IJITEE),Vol.5 Issue-5, Pp.`5-26 